Ingenium. Revista Electrónica de Pensamiento Moderno y Metodología en Historia de la Ideas ISSN-e 1989-3663

https://dx.doi.org/10.5209/inge.78431

\title{
El Barroco y lo barroco en la filosofía contemporánea: problemas y perspectivas
}

Paloma Martínez Matías ${ }^{1}$

Parece un hito obligado que los estudios que en la actualidad se dedican al barroco comiencen con un reconocimiento más o menos explícito del problema que José Lezama Lima expusiera en 1957 al inicio del texto «La curiosidad barroca». Allí llama la atención sobre la transformación que el término barroco habría sufrido desde el siglo XIX: si en él su sentido se limitaba a nombrar un estilo descrito como excesivo, poco profundo y otros calificativos por lo general peyorativos, en el siglo $\mathrm{XX}$, al pasar a valorarse como una manifestación estilística de relevancia en la esfera del arte, habría tenido lugar una extraordinaria ampliación de su significado que lo llevaría a abarcar producciones estéticas, científicas y filosóficas de naturaleza muy diversa, como «los ejercicios de Loyola, la pintura de Rembrandt y el Greco, las fiestas de Rubens y el ascetismo de Felipe de Champagne, la fuga bachiana, (...) la matemática de Leibniz, la ética de Spinoza». Incluso algún crítico proclive al mismo exceso atribuido a lo barroco -agrega Lezama Lima con cierta ironía- habría afirmado «que la tierra era clásica y el mar barroco», de suerte que los dilatados dominios de este concepto permitirían aludir a barrocos galerones hispanos que «recorren un mar teñido por una tinta igualmente barroca $»^{2}$. Por lo demás, el propio Lezama Lima semeja asumir la legitimidad de esta extensión del concepto de barroco al asignar tal condición a «los intentos de falansterio, de paraíso, hechos por los jesuitas en el Paraguay» ${ }^{3}$, conduciendo de este modo al término barroco a desbordar el campo de la estética y las creaciones culturales para situarlo en el de la intervención utópico-política.

Sin embargo, así como las consideraciones de Lezama Lima se inclinan a fijar el uso de la noción de barroco a fenómenos que se enmarcarían, con relativo grado de precisión, en los lindes del siglo XVII, la reflexión contemporánea no ha cesado de sobrepasarlos para conferir una hipotética condición barroca $-\mathrm{O}$, a veces, neobarroca- a expresiones artísticas, actitudes o perspectivas vigentes a día de hoy. Esta circunstancia ha abierto múltiples interrogantes, debates y controversias no sólo acerca de qué hechos, sucesos o fenómenos caerían bajo el alcance referencial del término barroco, sino también, y quizá ante todo, en torno a qué debe propiamente entenderse por este concepto, en qué consistiría su definición o cuáles serían los rasgos por los que cabría aplicarla a uno u otro fenómeno, dado que no otra cosa que la definición que se proporcione de la noción de barroco justificará la eventual determinación de ciertos hechos o fenómenos como barrocos.

En lo que concierne a este segundo aspecto de la cuestión -el que afectaría al contenido semántico de la palabra-, el panorama que ofrece la literatura sobre el barroco evidencia en este término una abundancia creciente de significados cuya disparidad impide la construcción de una definición unitaria y coherente del mismo. Lo cual vendría a poner en cuestión la posibilidad misma de otorgarle la categoría de concepto, al menos en la acepción tradicional de este vocablo como universal que estipula el conjunto de notas que, más allá de sus diferencias, han de cumplir todos aquellos objetos o fenómenos susceptibles de recibir el predicado «barroco». Así, este término se ha identificado con lo deforme, extraño e irregular; con una confluencia de perspectivas o gestos opuestos que, en ocasiones, se concreta en la simultánea exaltación de lo ultraterreno y lo terrenal; pero también, por el contrario, con un enaltecimiento de lo espiritual que aspiraría a superar lo material; con un tipo de ingenio cuya agudeza persigue proponer lo paradójico; con la glorificación del artificio y la profusión sin mesura del ornamento; con el gusto por el rodeo y lo laberíntico; con la emoción desbordante y el capricho o, en el otro extremo, con la plasmación de lo oceánico e infinito; con la actitud reverencial hacia lo inacabado y diferido... y todo ello no representa más que una muestra de lo que configuraría una larga lista de caracterizaciones que trascienden el terreno de la estética en la que la fragmentación se impone por encima de la unidad y la particularidad irreconciliable disuelve toda pretensión de identidad ${ }^{4}$.

En este horizonte de disenso y dispersión semántica se observa, no obstante, una cierta coincidencia en establecer en calidad de referente la delimitación cronológica que subyacía a las acotaciones de Lezama Lima. De manera que, en función de ella, se ha distinguido entre «el Barroco» como locución desig-

\footnotetext{
Paloma Martínez Matías es Profesora en el Departamento de Filosofía y Sociedad de la Universidad Complutense de Madrid. ORCID: orcid.org/0000-0003-2123-4087.

E-mail: palomamartinezm@filos.ucm.es

J. Lezama Lima, «La curiosidad barroca», en La expresión americana, Méjico, F.C.E., 1993, 79.

ibid., 85 .

Cf. W. Thayer, «El giro barroco. De G. Deleuze a W. Benjamin», Archivos: Revista de Filosofia, 2-3 (Dossier: Mímesis y política), 2007-8, 95-96.
} 
nativa del período histórico convergente a grandes trazos con el siglo XVII -o, en algunos casos, con la cultura forjada en esta época $-\mathrm{y}$ «lo barroco» como campo en el que se aglutinarían la vasta serie de rasgos, propiedades y peculiaridades que, desde mediados del XIX, se han ido asociando a este término en un largo proceso de adiciones, correctivos y disputas que sigue escamoteando la respuesta concluyente a la pregunta «qué es lo barroco» o en qué se cifraría la hipotética esencia de lo barroco. Por otra parte, ambas fórmulas se hallarían estrechamente engarzadas en su distinción, ya que el surgimiento de «lo barroco» como esfera de indagación y polémica sobre la posible conceptualización del término no obedece más que al interés que, desde el siglo XIX y hasta nuestros días, ha suscitado «el Barroco» como etapa histórica en vistas a la dilucidación tanto de la modernidad como de un presente que, pese oscilar entre la denominación de moderno y posmoderno, necesita seguir pensando la modernidad para alcanzar cierta lucidez sobre sí mismo 5 .

Los primeros trabajos que reivindican la importancia del Barroco, realizados por Heinrich Wölfflin $\mathrm{y}$, algo más tardíamente, por Werner Weisbach, se ven impulsados por la detección de ciertos aspectos de este período histórico que, a juicio de sus autores, habrían cobrado una nueva presencia en su contemporaneidad. Si las investigaciones de Wölfflin, focalizadas sobre la historia del arte, propugnan el regreso de rasgos estéticos específicos de las creaciones del siglo XVII -principalmente de la arquitectura que se lleva a cabo en Italia- en las manifestaciones artísticas de su época, los trabajos de Weisbach apuestan por dar razón de la emergencia de tales rasgos a partir de las circunstancias sociales, políticas e ideológicas que acompañan a la Contrarreforma, al tiempo que descansan sobre el vislumbre de una cierta afinidad entre ese momento convulso de la historia de Europa y la situación crítica que ésta atraviesa tras la Primera Guerra Mundial ${ }^{6}$. En esta estela exegética, destaca el texto de Walter Benjamin El origen del Trauerspiel alemán, escrito en 1925 y finalmente publicado en 1928. Por un lado, Benjamin apelará sin ambages a una actualidad del Barroco que funda sobre el parentesco que advierte entre la sensibilidad artística que reflejaría esta forma menor de la literatura del siglo XVII -y por eso tan desatendida como malinterpretada - y la que se desplegaría en las formas artísticas de las primeras décadas del siglo XX, especialmente en el expresionismo ${ }^{7}$. Pero, en contra de lo esperado, en este ensayo apenas se delinean los trazos generales que articularían el parentesco entre ambas sensibilidades artísticas y su objetivo tampoco residirá en explicitar en términos estéticos los elementos que las compondrían. Pues sus análisis constituyen ante todo una reflexión sobre el modo en que el Trauerspiel, a través de la alegoría como ley estilística del Barroco y armazón de su forma estética, «se asimila el material mediante el cual le nutren las condiciones históricas de su tiempo» ${ }^{8}$. O, dicho en otras palabras: para Benjamin el Trauerspiel vehicularía la comprensión que los hombres del siglo XVII poseían tanto del curso de la historia como de sus acciones en el seno de una realidad terrenal que, a causa de las nuevas perspectivas teóricas y científicas desarrolladas desde el Renacimiento y el doloroso cisma del cristianismo, se experimenta en ese momento como una realidad abandonada por la gracia divina.

Desde su concepción del siglo XVII como una época escindida y desgarrada por el conflicto entre las inquietudes religiosas y un mundo vaciado de todo sentido trascendente, Benjamin incide en la visión barroca de la historia como declive y decadencia, en la que el Trauerspiel enfatiza la vivencia de un tiempo homicida que condena a la criatura a la caducidad y la ruina, alegóricamente encarnadas en la imagen de la calavera9. No es de extrañar por ello que la melancolía aparezca en este escrito como una suerte de estado de ánimo epocal del Barroco que se liga al desconsuelo por una condición terrena en sí misma privada de la oportunidad de la redención ${ }^{10}$. La singular relevancia que adquiere en este escenario el vínculo entre el ideal de plena estabilización que instaura la Contrarreforma y la figura del soberano del absolutismo monárquico emplaza su principal tarea en la evitación de la catástrofe, equivalente a su habilidad para soslayar el decreto del estado de excepción $^{11}$. Pero la verdadera motivación de la investigación de Benjamin sobre el Barroco no llegaría a salir a la luz hasta sus textos más maduros, en los que la visión de la historia como ruina que registra en él se revela como aquella verdad de la que su propio tiempo ha de hacerse cargo en confrontación con la idea de progreso imperante en su historiografía: se trata de la verdad que descubre en la actualidad del siglo XX la misma catástrofe que el soberano barroco debía evitar y que aflora como resultado de una dilatada historia de dominación de unos seres humanos sobre otros que no ha cesado de producir muertos, escombros y ruinas ${ }^{12}$. Por eso, el Benjamin del Libro

\footnotetext{
Apoyándonos en esta distinción, de aquí en adelante sólo se aplicará el término «Barroco»-con inicio en mayúscula- para remitir al período histórico coincidente con el siglo XVII.

Cf. I. Iriarte, «Barroco contemporáneo», Orbis Tertius, XVII-18 (2012). Recuperado a partir de https://www.orbistertius.unlp.edu.ar/article/view/ OTV17n18a11.

Cf. W. Benjamin, El origen del Trauerspiel alemán, Madrid, Abada, 2012, 37-40

ibid., 221 .

Cf. ibid., 77 y ss.; cf. Thayer, «El giro barroco», 108

Cf. Benjamin, El origen del Trauerspiel alemán, 135 y ss.

Cf. ibid., 52 y ss.

Cf. W. Benjamin, «Sobre el concepto de historia», en Tesis sobre el concepto de historia y otros ensayos sobre historia y politica, Madrid, Alianza, $2021,71-72$.
} 
de los pasajes proclamará que el páthos de ese trabajo inconcluso anida en la tesis de que «no hay épocas de decadencia ${ }^{13}$ : bajo la mirada del historiador materialista, la presunta decadencia del Barroco se torna material rescatable para la eventual detención de un presente cuyo carácter catastrófico, encubierto por la ideología de los dominadores, amenaza con prolongarse indefinidamente y perpetuar el sufrimiento de la clase de los dominados y oprimidos.

Una voluntad análoga de traer el Barroco al presente -aunque cimentada sobre parámetros interpretativos muy distintos- se encuentra en textos como El pliegue. Leibniz y el Barroco, publicado en 1988. En él Gilles Deleuze localiza en Paul Klee, Fautrier, Dubuffet o Bettencourt a «los grandes pintores barrocos modernos» ${ }^{14}$, acusa en Le Corbusier un «espíritu barroco» ${ }^{15} \mathrm{y}$ hace de Mallarmé un «gran poeta barroco» ${ }^{16}$. Esta extensión del término barroco al mundo contemporáneo es solidaria de una original y compleja concepción del mismo como pliegue que se expande sin límite en un continuo de repliegues que englobarían tanto los repliegues infinitos de la materia como los pliegues igualmente infinitos del alma. Deleuze apunta de este modo a la irrupción en el Barroco de una nueva manera de entender la realidad que, al hilo de su acuñación de las nociones de mónada, armonía, curvatura o punto de inflexión, se exhibe en la metafísica y la matemática de Leibniz y logra su expresión plástica no sólo en la arquitectura del siglo XVII -con su escisión entre fachada e interior-, en los cuadros de Tintoretto y el Greco o en las esculturas de Bernini, sino también en creaciones del siglo XX. Esta nueva percepción de lo real que inaugura el Barroco conforma para Deleuze su respuesta a la crisis y derrumbe de la razón teológica que sustentaba el mundo medieval y que, tras el Renacimiento, se verá atacada por todos sus flancos, habiendo de enfrentarse a grietas y fisuras que únicamente podrán suturarse recurriendo al artificio ${ }^{17}$. Que esta operación se salde en la metafísica de Leibniz con una multiplicación de principios que, en su proliferación, tratan de seguir apuntalando la presencia de Dios en el orden general de lo creado -así como tras cada acontecimiento cuyo aparente sinsentido, unido al dolor que provoca en los seres humanos, delataría más bien su ausencia-, se debe a que el filósofo se ve impelido a erigirse en el abogado de Dios: los numerosos principios formulados por Leibniz se encaminan a reconstruir ese mundo antes penetrado por Dios que en el siglo XVII se derrumba, a justificar cada una de sus miserias, hasta el paroxismo que deriva en el postulado de que ese mundo devastado por laguerras de religión, la peste y las hambrunas, y cualquier otro que el curso de la historia depare a la humanidad, no es sino el mejor de los mundos posibles ${ }^{18}$.

Pero tras la crisis de la razón teológica llegará la crisis de lo que Deleuze llama la «razón humana» o ilustrada, sistematizada en la obra de Kant. Con ella se irá desplomando la confianza depositada en sus facultades y en su hipotética autonomía para sentar principios que garanticen el conocimiento universalmente válido, brinden directrices a la acción e instituyan normas para regular la vida social. A diferencia del episodio psicótico que, según Deleuze, supondría el Barroco ante la crisis de la razón teológica, la razón humana, que había creído poder suplantar a la razón teológica anclando en ella misma la fuente de un sentido inmanente a falta del trascendente, habrá de morir en esta segunda crisis por neurosis ${ }^{19}$. De ahí que, en paralelo con lo ocurrido en el siglo XVII, el renacimiento del espíritu barroco que impregnaría ciertas manifestaciones culturales del siglo XX sea leído por Deleuze como una nueva respuesta a la muerte de la razón, en este caso ilustrada: en tales manifestaciones se objetivarían tentativas «de salvar algo en ella o de reconstruirla $\rangle^{20}$ que explicarían el renovado aliento de lo barroco en su presente histórico.

En el contexto de esta problemática ocupan a su vez un lugar señalado tanto las interpretaciones del Barroco y lo barroco proyectadas sobre el mundo hispánico como las aportaciones a la misma efectuadas en él. Por un lado, se ha de tener en cuenta que la generalizada depreciación del Barroco desde consignas estéticas durante el siglo XVIII y buena parte del XIX convivía, en el caso de ciertos autores, con la valoración del siglo XVII español como la época de mayor esplendor artístico de su historia. Sobre esta base, la definición de Weisbach del barroco como arte de la Contrarreforma condujo a una asimilación no sólo del arte español con el más notorio exponente del Barroco, sino también de esta época con el paradigma de lo hispánico o con la esencia de lo español. Pero la bibliografía española sobre el Barroco ha contado con influencias no estrictamente coincidentes con el enfoque hermenéutico de Weisbach, aunque en ocasiones parcialmente afines a él. Entre ellas, la atracción por España que experimentarían autores representativos del romanticismo francés, que asociaban su imagen a la de una nación que habría permanecido al margen de los procesos civilizatorios promovidos por la Ilustración. Tal atracción, de la que se harían eco poetas como Paul Verlaine o Jean Moréas, acabaría desembocando, tras la estancia de Rubén Darío en París, en la ferviente recuperación de la poesía de Luis de Góngora de la Generación del 27 precisamente en atención a aquellos rasgos -formalismo,

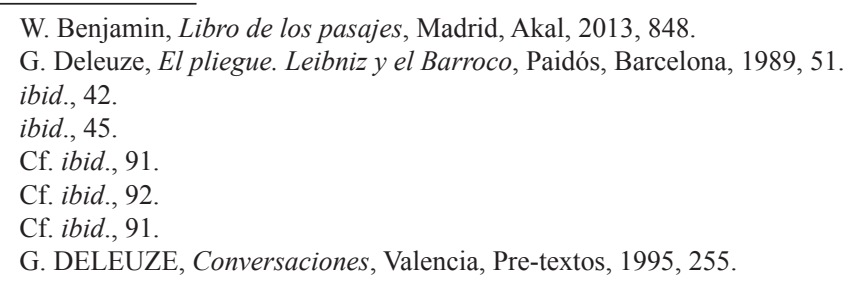


sensualismo, decadentismo o abandono a la fantasíapor los que hasta entonces había sido denostada ${ }^{21}$.

Con las ideas que se fraguan en este ambiente entroncan -si bien desde la distancia teórica- las reflexiones de Eugenio D'Ors de principios del siglo XX que culminan con la publicación, en 1935, del conjunto de ensayos recogidos bajo el título $\mathrm{Lo} \mathrm{Ba}$ rroco. Su novedad en ese momento radica en que D'Ors traspasa en ellos la aprehensión del Barroco como período histórico asumida por investigaciones precedentes para proponer una concepción de lo barroco como categoría supratemporal que lo ubicaría a lo largo de todo el curso de la historia y, por ende, en el ámbito de lo contemporáneo. Si D'Ors afronta el problema de obtener una «definición esencial de lo Barroco a través de la pluralidad específica de sus manifestaciones históricas y locales $\rangle^{22}$, su personal búsqueda le lleva a enunciar su conocida teoría del «eón», que promulga la vigencia histórica de dos concepciones de la vida, formas básicas de acción creadora o «estilos de cultura» ${ }^{23}$ opuestos: el «eón de lo clásico», vertebrado por conceptos como el orden, la razón, lo masculino, el lógos, la civilización o la regla, y el «eón de lo barroco», en el que se reunirían, como pares enfrentados a los anteriores, el caos, la pasión, lo femenino, el éthos, la barbarie o la excepción. Desde esta teoría, D'Ors divisará la impronta de lo barroco en artistas y literatos del siglo XVII -Gracián, Churriguera, Góngora-, pero también en filósofos como Rousseau, en figuras literarias como Robinson Crusoe, en la elipse de Kepler, en el carnaval o en el mito del Paraíso perdido. Pues tanto el eón de lo clásico como el de lo barroco serían «constantes históricas» ${ }^{24}$ que cohabitarían en constante tensión y cuya atribución a determinados fenómenos únicamente dependería de su mayor o menor prevalencia en ellos.

Más tardío, pero igualmente decisivo en la investigación española sobre el Barroco, es el trabajo de José Antonio Maravall La cultura del barroco. Análisis de una estructura histórica, de 1975. En contraste con la posición de D’Ors, Maravall retoma la línea interpretativa que circunscribe el término barroco a la designación del período histórico del siglo XVII con el fin de estudiar las circunstancias históricas -políticas, económicas, religiosas y sociales- que dieron lugar a las manifestaciones culturales y artísticas de esta época, con sus palmarias peculiaridades estilísticas. Por tanto, tal y como hace notar el título de su libro, con Maravall el concepto del barroco desborda de nuevo el terreno del arte y la estética para remitir a todos aquellos elementos que se dejan subsumir bajo la noción de cultura. Según sus análisis, centrados en el Barroco en España y en los que adopta tácitamente una óptica marxista, la situación de crisis económica y social, que involucra un factor de inestabilidad para la monarquía y las instituciones religiosas, origina una cultura orientada a reprimir todo conato de insurrección y resistencia frente al poder de las clases privilegiadas, así como a suscitar, mediante la penetración en las conciencias y el control psicológico, la conformidad de la población con el orden estableci$\mathrm{do}^{25}$.

En este sentido, Maravall perfila la cultura del Barroco como una «cultura dirigida», es decir, cuyo principal propósito estriba en la formación de una serie de dispositivos culturales al servicio de un ejercicio de poder ideológico con el que las clases dominantes buscan el sometimiento consentido de las dominadas. Asimismo, como una cultura diseñada para las masas, urbana y claramente conservadora, en la que la exaltación y fomento de la novedad en la esfera artística será utilizada como medio de encubrimiento de la inmutabilidad del orden político-social. En función de ello, Maravall subraya la presencia en el Barroco de un ingrediente kitsch, propio de una cultura de masas vulgarizada, proclive a la generación de tipos y a la repetición estandarizada de patrones y que, consumida de manera irreflexiva, promovería el conservadurismo social ${ }^{26}$. No otro que este ingrediente kitsch le induce a afirmar la existencia de un «nexo entre procedimientos del Barroco y de nuestros días $»^{27}$ por el que, a pesar de la limitación histórica que Maravall impone a este concepto, su investigación no dejará de lanzarlo hacia el presente.

De maneras dispares, la reflexión sobre el Barroco en Latinoamérica entra en diálogo y a la vez en disputa con las posiciones de D'Ors y Maravall para, entre otras metas, argumentar sobre la singularidad de su despliegue tanto en la América colonial como, posteriormente, en el arte y la literatura contemporáneos producidos en el continente americano. Parte de tal reflexión gira en torno a la implantación de las formas artísticas del Barroco español -sobre todo en la arquitectura y la pintura- en los territorios colonizados. Si se admiten las perspectivas de Weisbach y Maravall, ésta no sólo estaría motivada por la pretensión de introducir a los nativos en la cultura hispánica, sino también por la de consolidar su aceptación del poder imperial a través de la promoción de la fe religiosa -tarea que se intensifica con la Contrarreforma- y evitar cualquier forma de heterodoxia con respecto al dogma católico. Sin embargo, el mero hecho de que los artífices de las creaciones plásticas y arquitectónicas destinadas a fines religiosos fueran indígenas conllevó la incorporación de elementos

\footnotetext{
Cf. Iriarte, «Barroco contemporáneo» y O. Cornago Bernal, «Nuevos enfoques sobre el Barroco y la (Pos)Modernidad (a propósito de dos estudios de Fernando R. de la Flor)», Dicenda, Cuadernos de Filología Hispánica, 22 (2004), 30.

E. d'Ors, Lo Barroco, Madrid, Tecnos, 1993, 69.

ibid., 74.

ibid., 62.

Cf. J. A. Maravall, La cultura del barroco. Análisis de una estructura histórica, Barcelona, Ariel, 1975, 112.

Cf. ibid., 197.

ibid., 269
} 
pictóricos prehispánicos que, paulatinamente, provocaron un verdadero sincretismo o simbiosis entre las formas barrocas y las autóctonas ${ }^{28}$.

Por la naturaleza sin duda híbrida de estas creaciones, pero dotada de una extrema originalidad, en su ya citado texto «La curiosidad barroca» Lezama Lima defiende la gestación durante la época colonial de un barroco americano de rasgos antitéticos al europeo que lo proveerían de un carácter emancipador: tensión -fruto de la mixtura-, plutonismo -término que parece aludir a una especie de temeridad rebelde que rompe con lo esperado y reduce a fragmentos lo heredado para recomponerlo en una nueva unidad-y plenitud inversa a la degeneración que asigna al barroco europeo ${ }^{29}$. Contra la equivalencia del barroco con el arte de la Contrarreforma planteada por Weisbach, Lezama Lima sostiene que el barroco del Nuevo Mundo se forja como un arte de la «contraconquista ${ }^{30}$. A un tiempo, que «el primer americano que va surgiendo dominador de sus caudales es nuestro señor barroco ${ }^{31}$. Un señor que, al contrario que el hombre barroco europeo, sería amigo de la Ilustración y cuyo espíritu rebelde le dirigiría hacia la libertad y la autonomía ${ }^{32}$. Con ello, la mirada hacia el pasado de la época barroca deviene en Lezama Lima un instrumento para la construcción de una identidad nacional que, sin renegar plenamente de la etapa colonial, la somete a una relectura que reivindica la invención latinoamericana de una forma de estar en el mundo - un «vivir completo» ${ }^{33}$, dice el escritor cubano- que debe proseguir su camino hacia una independencia aún percibida como incompleta.

Pero es el también cubano Severo Sarduy quien, reconociendo su deuda con el legado de Lezama Lima aunque bajo el influjo de autores como Roland Barthes y Jacques Lacan, tal vez más ha contribuido a la delimitación de un barroco contemporáneo del que quiere desprenderse la definición de un presente neobarroco. En su primer ensayo sobre esta temática, «El barroco y el neobarroco», de 1972, Sarduy se decanta, ante la dispersión semántica del término barroco, por una restricción del mismo que, acotándolo de nuevo al terreno de la estética, codifique la «pertinencia de su aplicación al arte latinoamericano actual» ${ }^{34}$. No obstante, su rechazo a la identificación de d'Ors de lo barroco con lo primitivo y con la nostalgia del Paraíso perdido, a partir del cual Sarduy emplaza el «festín barroco» en la «apoteosis del artificio» ${ }^{35}$ y la parodia, encauzará sus consideraciones en el curso de este ensayo hacia un terreno que, una vez más, sobrepasa la esfera del arte. Para Sarduy, artificio y parodia se ensamblan con la superabundancia y el desperdicio, que hacen patente en lo barroco un horror vacui sobre el que reposaría su incansable inclinación a la proliferación y la saturación sin límites. Pero, puesto que éstas entrañan por fuerza la disarmonía, la falta de homogeneidad y el desequilibrio, en su expresión en el arte latinoamericano actual Sarduy interpretará un signo de la «carencia que constituye nuestro fundamento epistémico» ${ }^{36}$. O, lo que es lo mismo, del desvelamiento en su presente de la falta de un lógos ordenador, capaz de procurar un saber definitivo y cerrado tanto sobre el mundo como sobre nosotros mismos, falta con la que se abriría el escenario del destronamiento de la razón y la discusión.

Dos años después Sarduy publica el libro Barroco para ampliar el foco de su indagación sobre este tema y reiterarse en las conclusiones de su primer ensayo. Al examinar en esta ocasión el Barroco histórico, Sarduy señala que el tránsito del círculo a la elipse que implica la irrupción de la cosmología de Kepler -ya calificada por D'Ors como barroca-, tránsito en el que la imagen de perfección y centralidad del círculo es sustituida por la del descentramiento y la deformidad, encarnaría el paso de lo clásico a lo barroco. Lo barroco quedará además anudado a la idea de infinitud, en la que resuena asimismo el horror vacui subyacente a la proliferación desbordante de lo barroco, que se deduce de la órbita elíptica. Por ello, Sarduy situará en ésta la clave que permite descifrar la particularidad de las manifestaciones del Barroco, en las que trasluciría el descentramiento de la elipse a través de la puesta en juego de un doble centro virtual o real -en el Greco y en Rubens- o que plasmarían sus consecuencias por medio de la elipsis del sujeto -en Velázquez y Cervantes-. Por otra parte, el retorno que observa en el arte contemporáneo -aunque no exclusivamente contemporáneo- de estos recursos barrocos le incita a preguntarse, en el «Suplemento» con el que cierra su libro, qué significaría a día de hoy la práctica del barroco.

En su respuesta, que abrirá nuevas líneas de pensamiento sobre el Barroco y lo barroco, éste aparece como una instancia que, al embestir con su desmesura contra la economía del lenguaje y sus intenciones meramente comunicativas, comporta al mismo tiempo un ataque contra la lógica capitalista de la producción rentable, el consumo, la acumulación y la ganancia. Pues al «malgastar, dilapidar, derrochar lenguaje» en su culto por la superabundancia y el desperdicio, la práctica del barroco operaría sin más finalidad que la acción gratuita e innecesaria, impugnando el «orden

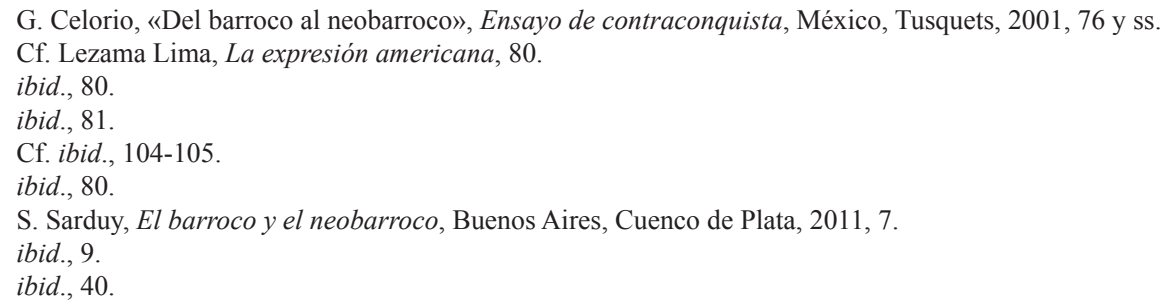


supuestamente normal de las cosas» y su sujeción a la tiranía de la utilidad ${ }^{37}$. Así, en el enfoque de Sarduy lo barroco se da a ver como una dimensión que, nacida en el seno de la propia modernidad al modo de una suerte de excrecencia indeseada que la alejaría de sus principios, también cabría hacer valer como un elemento de crítica del proyecto moderno allí donde éste muestra sus más esenciales fallas: en su tenaz ocultación de los efectos perversos del seguimiento ciego e incondicionado de tales principios; en los espacios en los que, a través del trabajo, el olvido del juego y el placer, la reducción de la razón a puro cálculo y la imposición de un centro incuestionado, la modernidad ilustrada atenta contra la misma libertad que persigue.

$* * * * * * * * * * * * * * * * * * * * * *$

Las problemáticas a esclarecer en torno a la cuestión del Barroco y lo barroco, al igual que los autores que se han ocupado de ellas, exceden sin duda lo expuesto en este breve trayecto. Sin ánimo de agotarlas, la sección monográfica que con él se introduce desea suponer una contribución relevante al debate actual sobre este tema al cubrir, con los artículos que lo integran, un espectro de variantes en el que se visibiliza tanto su riqueza como su importancia en el marco de la reflexión filosófica contemporánea.

En primer término, el trabajo de Marcia Martínez García «La cuestión de los milagros en el pensamiento leibniziano, frente al determinismo absoluto y el voluntarismo teológico» se instala en el terreno de la filosofía del siglo XVII para estudiar el problema de la difícil tarea a la que se enfrenta el pensamiento de Leibniz en su afán por conjugar, por un lado, la plena confianza racionalista en el poder de la razón y el determinismo ontológico que se deriva de ella y, por otro, el cristianismo, cuya moral rechaza la idea de determinación en función del dogma de la libre voluntad del hombre y de la acción divina. El artículo explica la forzosa reformulación de las categorías modales de necesidad y contingencia acometida por Leibniz a fin de salvaguardar la libertad de Dios. Pero su pretensión de justificar, contra la posición de Spinoza, la existencia de lo milagroso como producto de una volición extraordinaria de Dios le hará recurrir a distinciones conceptuales que restan coherencia al conjunto de su filosofía y que pondrían en peligro un peligro del cual Leibniz no habría dejado de ser consciente- la epistemología racionalista.

También desde la esfera de la filosofía del Barroco, Claudiu Marius Mesaroș, en su artículo «Jesuits, Transylvanian baroque and the Middle Ages: Ignatius Batthyány and Saint Gerardus of Cenad», se adentra en el despliegue en Europa Central de la llamada «escolástica barroca», designación para una cultura filosófica que, si bien se desarrolla en la época moderna -principalmente entre los siglos XV y
XVII, aunque en algunos enclaves pervive hasta finales del XVIII-, configura una forma de pensamiento antimoderno que, nutriéndose aún del aristotelismo, se caracteriza por sus lazos con las instituciones religiosas y por su adhesión al ideal jesuita de la educación, que propugnaba la construcción de bibliotecas dotadas de una torre de observación astronómica. En este contexto se ahonda en la figura de Ignatius Batthyány, obispo de Transilvania e intelectual barroco que aspiraba a establecer una íntima conexión entre la ciencia, el arte, la arquitectura y la religión y que, además de construir el centro de aprendizaje Alba Iulia siguiendo el modelo jesuita, edita el legado, en parte filosófico, del obispo benedictino del siglo XI Gerardus de Cenad.

Los autores del artículo «Una ontología de la monstruosidad americana. Del monstruo fabuloso al monstruo ontológico», Bernat Castany Prado y Christian Snoey Abadías, abordan en él el asunto de las repercusiones del descubrimiento del continente americano en el interés por los monstruos y lo monstruoso de la Europa de los siglos XVI y XVII, agudizado en comparación con la época medieval como efecto paradójico del surgimiento de la ciencia y el racionalismo. Aun cuando esta cuestión será dirimida en el Nuevo Mundo a partir de las formas de comprensión de lo monstruoso ya vigentes antes de su descubrimiento, según sus autores lo tipificado como tal termina por adquirir una significación ontológica al extenderse no sólo al continente americano, sino al mundo en su totalidad: ambos serán contemplados como realidades monstruosas que causarían terror en la medida en que el descubrimiento de América traerá consigo la emergencia de la imagen, tan inquietante como angustiosa, de un mundo infinito e indefinido que desafía las facultades cognoscitivas del ser humano.

Atendiendo a la importancia del retrato tanto en la obra de Velázquez como en el naturalismo barroco, el ensayo de Ana Maqueda de la Peña «El retrato en Las meninas» se apoya en las apreciaciones de autores barrocos o que han investigado esta época histórica -como Gracián, Benjamin, Deleuze o Maravallpara explorar las dimensiones diversas que se abren al analizar la singularidad que la técnica del retrato exhibe en esta obra. Su descripción se lleva a término a través de un recorrido por una serie de motivos que la autora utiliza como espacio de reflexión sobre el Barroco y su pintura. Entre ellos, la cuestión de la mirada y el contraste entre la del propio Velázquez en el espejo y la del resto de figuras del cuadro, que dirigen su mirada hacia el exterior interpelando a un espectador que entonces queda convertido en una suerte de enviado de la posteridad para la que posan. La alegoría como lugar de conflicto entre lo caduco y lo eterno, vinculada a la imagen distante y opaca de los monarcas que parecen resignarse a su propia mortalidad. O la referencia al teatro que evoca la es- 
cenificación del cuadro, que expresaría la metáfora barroca de la vida como representación teatral.

Por último, centrándose en el campo de la política, el ensayo de Roberto Navarrete Alonso «Soberanía, dictadura y barroco. En torno a Walter Benjamin y Carl Schmitt» analiza la relación entre el texto de Benjamin El origen del Trauerspiel alemán y la obra del jurista y teórico del Estado Carl Schmitt, de la que dan noticia no sólo las referencias que figuran en ese texto a la Teología política de Schmitt, sino también la carta que Benjamin le escribiera en 1930, ambas ocultadas en las primeras ediciones del legado benjaminiano. Con toda probabilidad, semejante ocultación se debió a la voluntad de resguardar de la luz pública cualquier posible convergencia entre la visión de Benjamin de la política y la defendida por Schmitt, durante años miembro prominente del partido nacionalsocialista. Pero en este artículo se argumenta que si bien la asociación entre los conceptos de dictadura, soberanía y estado de excepción que se plantea en El origen del Trauerspiel alemán procedería del libro de Schmitt $L a$ dictadura, las anotaciones de este último sobre este trabajo de Benjamin evidencian la distancia que, en la evolución de su pensamiento, Schmitt percibe entre su propia concepción de la política y la allí plasmada a propósito del soberano barroco.

Esta sección monográfica dedicada al Barroco se cierra con una reseña del libro de Frédéric Lenoir, El milagro de Spinoza. Una filosofia para iluminar nuestra vida, a cargo de Sergio Casado Chamizo. Un libro que constituye una entusiasta invitación a la lectura de Spinoza que, alejándose de las interpretaciones al uso, no dejará de resaltar, a tenor del problema spinoziano de la armonización de lo racional y lo afectivo, la validez de su filosofía para entender la realidad de lo humano.

\section{Bibliografía}

Benjamin, Walter (2012): El origen del Trauerspiel alemán, trad. de A. Brotons Muñoz, Madrid, Abada.

Benjamin, Walter (2021): «Sobre el concepto de historia», en Tesis sobre el concepto de historia y otros ensayos sobre historia y política, trad. de J. Maiso y J. A. Zamora, Madrid, Alianza.

Benjamin, Walter (2013): Libro de los pasajes, ed. de Rolf Tiedemann, Madrid, Akal.

Celorio, Gonzalo (2001): «Del barroco al neobarroco», Ensayo de contraconquista, México, Tusquets, 73-105.

Cornago Bernal, Óscar (2004): «Nuevos enfoques sobre el Barroco y la (Pos)Modernidad (a propósito de dos estudios de Fernando R. de la Flor)», Dicenda, Cuadernos de Filología Hispánica, 22, 27-51.

Deleuze, Gilles (1989): El pliegue. Leibniz y el Barroco, trad. de J. Vázquez y U. Larraceleta, Paidós, Barcelona.

Deleuze, Gilles (1995): Conversaciones, trad. de J. L. Pardo, Valencia, Pre-textos.

D’Ors, Eugenio (1993): Lo Barroco, Madrid, Tecnos.

Iriarte, Ignacio (2012): «Barroco contemporáneo», Orbis Tertius, XVII-18. Recuperado a partir de https://www.orbistertius.unlp.edu.ar/article/view/OTv17n18a11.

Lezama Lima, José (1993): «La curiosidad barroca», en La expresión americana, Méjico, F.C.E., 79-106.

Maravall, José Antonio (1975): La cultura del barroco. Análisis de una estructura histórica, Barcelona, Ariel.

Sarduy, Severo (2011): El barroco y el neobarroco. Apostillas por Valentín Díaz, Buenos Aires, Cuenco de Plata.

Sarduy, Severo (1987): «Barroco», en Ensayos generales sobre el barroco, Buenos Aires, F. C. E., 147-212.

Thayer, Willy (2007-8): «El giro barroco. De G. Deleuze a W. Benjamin», Archivos: Revista de Filosofía, 2/3 (Dossier: Mímesis y política), 93-119. 